Dhaka Univ. J. Sci. 61(2): 167-171, 2013 (July)

\title{
A One Pot Synthesis of 5, 7-diaryl-1,5-dihydro (or 1, 2, 3, 5-tetrahydro)- pyrano[2, 3-D] pyrimidin-2, 4-diones (or 2-thioxo-4-ones)
}

\author{
M. Mahbubur Rahman ${ }^{1}$, S. Mosaddeq Ahmed ${ }^{2}$, S. M. A. Hakim Siddiki ${ }^{3}$, Md. Ershad Halim ${ }^{1}$, \\ Kawsari Akhter ${ }^{1}$, M. Giasuddin Ahmed ${ }^{1}$ and U. K. R. Romman ${ }^{1 *}$ \\ ${ }^{1}$ Department of Chemistry, Dhaka University, Dhaka-1000, Bangladesh \\ ${ }^{2}$ Department of Natural Science, American International University-Bangladesh (AIUB), Banani, Dhaka-1213, Bangladesh. \\ ${ }^{3}$ Graduate School of Material Science, University of Hyogo, 678-1297, Japan
}

(Received : 1 August 2012; Accepted : 3 December 2012)

\begin{abstract}
A number of 5, 7-diaryl-1,5-dihydro (or 1, 2, 3, 5-tetrahydro)- pyrano[2, 3- $d$ ] pyrimidin-2, 4-diones (or 2-thioxo-4-ones) (3a-f) have been synthesized in one-step by cyclocondensation of barbituric acid or thiobarbituric acid (1) with arylideneacetophenones (2a-c), in glacial acetic acid in the presence of phosphorous pentoxide. The structures of the compounds 3a-f have been determined by $\mathrm{UV}$, IR, ${ }^{1} \mathrm{H} \mathrm{NMR},{ }^{13} \mathrm{C}$ NMR, mass spectral data and elemental analyses.
\end{abstract}

Keywords: arylideneacetophenone, barbituric acid, thiobarbituric acid, cyclocondensation.

\section{Introduction}

Synthesis of pyranopyrimidines has been an interesting work because of the pharmacological activities ${ }^{1-4}$ associated with this system. A variety of routes ${ }^{5-8}$ for the synthesis of these compounds have been reported, but the majority of them involve a number of steps and the yields are relatively poor. This initiated to develop an efficient method for the synthesis of these compounds in better yields. There is a report ${ }^{9}$ on the reactions of barbituric acids with $\alpha, \beta$-unsaturated carbonyl systems.

Having this background, in continuation of the reported works $^{10,11}$ on the synthesis of 5, 7-diaryl-1, 5-dihydropyrano[2,3- $d$ pyrimidin-2, 4-diones, we report herein syntheses of 5-(4-chloro-phenyl)-7-phenyl-1, 5-dihydropyrano[2, 3-d]pyrimidine-2, 4-dione 3a, 5-(4-chlorophenyl)-7-p-tolyl-1, 5-dihydro-pyrano[2, 3- $d]$ pyrimidine2,4-dione 3b, 5-(4-chloro-phenyl)-7-phenyl-2-thioxo-1, 2, 3, 5-tetrahydro-pyrano[2, 3- $d$ ]pyrimidine-4-one 3c, 5-(4chloro-phenyl)-2-thioxo-7-p-tolyl-1，2，3，5-tetrahydropyrano[2, 3- $d$ ]pyrimidine-4-one 3d, 5-(4-chloro-phenyl)7-(4-nitro-phenyl)-1, 5-dihydro-pyrano[2, 3- $d$ ]pyrimidine2, 4-dione 3e and 5-(4-chloro-phenyl)-7-(4-nitro-phenyl)2-thioxo-1, 2, 3, 5-tetrahydro-pyrano[2, 3-d]pyrimidin-4one $3 \mathbf{f}$ by selecting a number of arylideneacetophenones (2a-c) as the $\alpha, \beta$-unsaturated carbonyl system having different substituents on the aromatic rings for reaction with barbituric acid or thiobarbituric acid (1) as the active methylene component.

The Compounds 3a-f have been characterized by different spectroscopic methods and elemental analyses. The formation of compounds 3a-f may be explained by the

\footnotetext{
*Author for Correspondence, e-mail: uromman@yahoo.com
}

initial formation of a 1:1 adduct (A) followed by cyclocondensation (Scheme 1). The formation of such an adduct has been reported ${ }^{12}$ in the literature.

\section{Experimental}

The UV spectra were run in methanol using SHIMADZU-UV$160 \mathrm{~A}$ ultraviolet spectrophotometer with a scanning range of $800-200 \mathrm{~nm}$ using methanol as solvent. The IR spectra were recorded as $\mathrm{KBr}$ pellet using SHIMADZU FT-IR 8400S infrared spectrophotometer in the range of $4000-400 \mathrm{~cm}^{-1}$. The ${ }^{1} \mathrm{H}-$ and ${ }^{13} \mathrm{C}$ - NMR spectra were recorded on $600 \mathrm{MHz}$ NMR spectrometer. The solvent used was $\mathrm{d}_{6^{-}}$DMSO and TMS is being used as a reference. All the compounds gave expected $\mathrm{C}, \mathrm{H}$ and $\mathrm{N}$ analyses.

3-(4-chloro-phenyl)-1-phenyl propenone 2a, 3-(4-chlorophenyl)-1-p-tolyl-propenone $2 \mathbf{b}$ and 3-(4-chloro-phenyl)-1-(4nitro-phenyl)-propenone 2c were prepared from the reactions of corresponding substituted aldehydes and substituted acetophenones by following primarily literature method ${ }^{13}$ with modification of the reaction conditions wherever necessary. The reactions described in the present paper were carried out following a general procedure. ${ }^{9}$

General Procedure: A mixture of arylideneacetophenone $(0.005 \mathrm{~mol})$ and barbituric acid or thiobarbituric acid $(0.005$ mol) were dissolved in acetic acid $(10 \mathrm{~mL})$ and $\mathrm{P}_{2} \mathrm{O}_{5}(2 \mathrm{~g})$ in a round-bottomed flask equipped with a magnetic stirrer, a refluxing condenser and a drying tube. The reaction mixture was refluxed at $135-140^{\circ} \mathrm{C}$ for $6-8.5$ hours and the course of the reaction was followed by TLC on silica gel plates (eluting solvent; EtOAc: $\mathrm{CHCl}_{3}=3: 2$ ). The mixture was allowed to cool and treated with crushed ice. The solid, thus obtained, was filtered off, washed with cold water, dried and purified by recrystallization from rectified spirit. 
Scheme-1<smiles></smiles>

1

\begin{tabular}{c} 
Produc \\
\hline $3 a$ \\
$3 b$ \\
$3 c$ \\
$3 d$ \\
$3 e$ \\
$3 f$
\end{tabular}

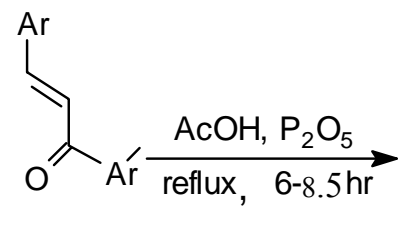

2<smiles></smiles>

A<smiles>CCO</smiles><smiles></smiles>

(1:1 ratio)

\section{Results and Discussion}

Compounds 3a-f have been synthesized from 1 and the corresponding 2a-c in presence of glacial acetic acid and $\mathrm{P}_{2} \mathrm{O}_{5}$ under refluxing conditions in an analogous manner reported previously ${ }^{9}$. The assignment to the structures of the compounds 3a-f was made on the basis of their UV, IR, ${ }^{1} \mathrm{H}$ $\mathrm{NMR},{ }^{13} \mathrm{C} \mathrm{NMR}$, mass and elemental analyses.

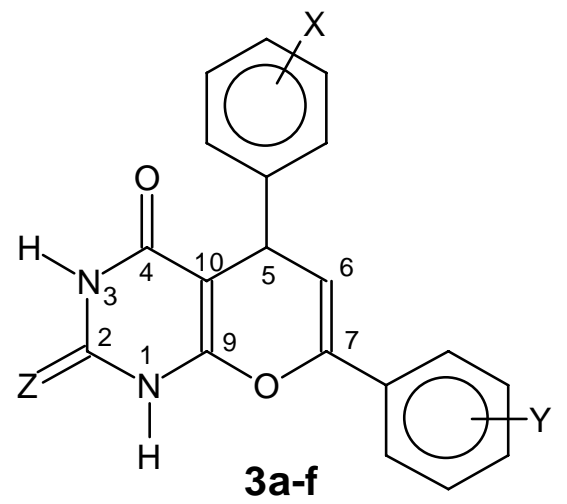

\begin{tabular}{ccccccc}
\hline Substituent & 3a & 3b & 3c & 3d & 3e & 3f \\
\hline $\mathbf{X}$ & $4-\mathrm{Cl}$ & $4-\mathrm{Cl}$ & $4-\mathrm{Cl}$ & $4-\mathrm{Cl}$ & $4-\mathrm{Cl}$ & $4-\mathrm{Cl}$ \\
$\mathbf{Y}$ & $\mathrm{H}$ & $4-\mathrm{CH}_{3}$ & $\mathrm{H}$ & $4-\mathrm{CH}_{3}$ & $4-\mathrm{NO}_{2}$ & $4-\mathrm{NO}_{2}$ \\
$\mathbf{Z}$ & $\mathrm{O}$ & $\mathrm{O}$ & $\mathrm{S}$ & $\mathrm{S}$ & $\mathrm{O}$ & $\mathrm{S}$ \\
\hline
\end{tabular}

In their UV spectra of compounds 3a-f the observed $\lambda_{\max }$ values agree well to the expected values. The absorption bands in the range $312-286 \mathrm{~nm}$ may be assigned to the $\pi \rightarrow \pi^{*}$ of $\mathrm{C}=\mathrm{O}$ in these compounds. The weak $\mathrm{n} \rightarrow \pi^{*}$ absorption bands in the cases of these compounds due to
$\mathrm{C}=\mathrm{O}$ were probably masked within the $\pi \rightarrow \pi^{*}$ absorption range of 312-286 $\mathrm{nm}$.

The IR data of the compounds 3a-f (Table 2) showed sharp as well as broad bands in the range $\left(v_{\max }\right) 3476-3100 \mathrm{~cm}^{-1}$ indicating the presence of $\mathrm{N}-\mathrm{H}$ group. The absorption bands 
at $1759-1655 \mathrm{~cm}^{-1}$ indicate the presence of non-conjugated $\mathrm{C}=\mathrm{O}$ stretching including the barbituric acid moieties. ${ }^{14}$ The bands at $1606-1514 \mathrm{~cm}^{-1}$ were assigned to $\mathrm{C}=\mathrm{C}$ of aromatic rings and $\mathrm{C}=\mathrm{N}$ of the conjugated form of barbituric acid part. Additional bands were observed at $1451-813 \mathrm{~cm}^{-1}$ due to these structural units. ${ }^{14}$

Table 1. Reaction conditions and analytical data of the compounds 3a-f.

\begin{tabular}{|c|c|c|c|c|c|c|c|}
\hline Compound & $\begin{array}{l}\text { Reflux } \\
\text { time (hr) }\end{array}$ & $\begin{array}{l}\text { Reaction } \\
\text { temp. }\left({ }^{0} \mathrm{C}\right)\end{array}$ & $\begin{array}{l}\% \mathrm{C} \\
\text { Found } \\
\text { (Calcd) }\end{array}$ & $\begin{array}{l}\% \mathrm{H} \\
\text { Found } \\
\text { (Calcd) }\end{array}$ & $\begin{array}{l}\% \mathrm{~N} \\
\text { Found } \\
\text { (Calcd) }\end{array}$ & Mol. formula & $\operatorname{MS}(\mathrm{m} / \mathrm{z})$ \\
\hline $3 a$ & 8 & 135 & $\begin{array}{l}64.05 \\
(64.69)\end{array}$ & $3.65(3.71)$ & 7.05 (7.94) & $\mathrm{C}_{19} \mathrm{H}_{13} \mathrm{~N}_{2} \mathrm{O}_{3} \mathrm{Cl}$ & 352.5 \\
\hline $3 b$ & 8.5 & 140 & $\begin{array}{l}64.32 \\
(65.49)\end{array}$ & $4.39(4.12)$ & $7.32(7.64)$ & $\mathrm{C}_{20} \mathrm{H}_{15} \mathrm{~N}_{2} \mathrm{O}_{3} \mathrm{Cl}$ & 366.5 \\
\hline $3 c$ & 7 & 135 & $\begin{array}{l}63.49 \\
(64.21)\end{array}$ & $4.35(4.15)$ & $7.80(7.85)$ & $\mathrm{C}_{19} \mathrm{H}_{13} \mathrm{~N}_{2} \mathrm{O}_{2} \mathrm{Cl} \mathrm{S}$ & 368.5 \\
\hline $3 d$ & 6.5 & 138 & $\begin{array}{l}63.56 \\
(62.74)\end{array}$ & $4.30(4.10)$ & $7.56(7.32)$ & $\mathrm{C}_{20} \mathrm{H}_{15} \mathrm{~N}_{2} \mathrm{O}_{2} \mathrm{Cl} \mathrm{S}$ & 382.5 \\
\hline $3 e$ & 6 & 140 & $\begin{array}{l}58.48 \\
(57.37)\end{array}$ & $3.15(3.04)$ & $10.15(10.56)$ & $\mathrm{C}_{19} \mathrm{H}_{12} \mathrm{~N}_{3} \mathrm{O}_{5} \mathrm{Cl}$ & 397.5 \\
\hline $3 f$ & 6 & 143 & $\begin{array}{l}58.75 \\
(58.15)\end{array}$ & $3.25(3.15)$ & $10.24(10.30)$ & $\mathrm{C}_{19} \mathrm{H}_{12} \mathrm{~N}_{3} \mathrm{O}_{4} \mathrm{Cl} \mathrm{S}$ & 413.5 \\
\hline
\end{tabular}

Table 2. Physical Constants, IR and UV of compounds 3a-f.

\begin{tabular}{|c|c|c|c|c|c|c|c|c|}
\hline \multirow{3}{*}{ Compound } & \multirow{3}{*}{$\begin{array}{l}\text { m.p. } \\
\left({ }^{0} \mathrm{C}\right)\end{array}$} & \multirow{3}{*}{$\begin{array}{l}\text { Yield } \\
(\%)\end{array}$} & \multirow{3}{*}{$\begin{array}{l}\mathrm{R}_{\mathrm{f}} \text { value } \\
\text { (eluting solvents) }\end{array}$} & \multicolumn{4}{|c|}{$\mathrm{IR}, v_{\max }$ in $\mathrm{cm}^{-1}$} & \multirow[b]{2}{*}{$\begin{array}{l}\mathrm{UV}, \lambda_{\max } \\
(\mathrm{nm})(\varepsilon)\end{array}$} \\
\hline & & & & \multirow[t]{2}{*}{$\mathrm{N}-\mathrm{H}$} & \multirow{2}{*}{$\begin{array}{l}\mathrm{C}=\mathrm{O} \\
\text { non- } \\
\text { conj. }\end{array}$} & \multirow{2}{*}{$\begin{array}{l}\mathrm{C}=\mathrm{O} \\
\text { arom, C- } \\
\quad \mathrm{N}\end{array}$} & \multirow[t]{2}{*}{$\begin{array}{l}\mathrm{C}=\mathrm{C} \\
\text { (arom. \& bar. } \\
\text { acid moieties) }\end{array}$} & \\
\hline & & & & & & & & $\pi \rightarrow \pi^{*}, \mathrm{n} \rightarrow \pi^{*}$ \\
\hline $3 a$ & $312-314$ & 58 & $\begin{array}{c}0.33 \\
\left(\text { EtOAc: } \mathrm{CHCl}_{3}=3: 2\right)\end{array}$ & 3230 & $\begin{array}{l}1759 \\
1718\end{array}$ & $\begin{array}{l}1603 \\
1514\end{array}$ & $\begin{array}{c}1417,1240 \\
1028,819\end{array}$ & $\begin{array}{c}286 \\
(3780)\end{array}$ \\
\hline $3 b$ & $141-142$ & 77 & $\begin{array}{c}0.71 \\
\left(\text { EtOAc: } \mathrm{CHCl}_{3}=3: 2\right)\end{array}$ & 3474 & 1655 & 1599 & $1332,1012,813$ & $\begin{array}{c}312 \\
(25994)\end{array}$ \\
\hline $3 c$ & $310-312$ & 70 & $\begin{array}{c}0.81 \\
\text { (EtOAc: } \mathrm{CH}_{3} \mathrm{OH}=3: 2 \text { ) }\end{array}$ & 2857 & 1680 & $\begin{array}{l}1606 \\
1563\end{array}$ & $\begin{array}{c}1451,1220, \\
1110,1050,827\end{array}$ & $\begin{array}{c}286 \\
(3925)\end{array}$ \\
\hline $3 d$ & $281-284$ & 85 & $\begin{array}{c}0.41 \\
\left(\text { EtOAc: } \mathrm{CHCl}_{3}=3: 2\right)\end{array}$ & 3046 & 1680 & $\begin{array}{l}1600 \\
1558\end{array}$ & $1450,1120,815$ & $\begin{array}{c}286 \\
(5078)\end{array}$ \\
\hline $3 e$ & $298-300$ & 76 & $\begin{array}{c}0.32 \\
\left(\text { EtOAc: } \mathrm{CHCl}_{3}=4: 1\right)\end{array}$ & 3290 & $\begin{array}{l}1726 \\
1683\end{array}$ & $\begin{array}{l}1607 \\
1518\end{array}$ & $\begin{array}{c}1437,1348 \\
1286,1101,856\end{array}$ & $\begin{array}{c}301 \\
(2475)\end{array}$ \\
\hline $3 f$ & $126-128$ & 52 & $\begin{array}{c}0.58 \\
\left.\text { (EtOAc: } \mathrm{CHCl}_{3}=4: 1\right)\end{array}$ & 3476 & 1681 & 1583 & $\begin{array}{c}1347,1138 \\
854\end{array}$ & $\begin{array}{c}317 \\
(10453)\end{array}$ \\
\hline
\end{tabular}

The N-H protons at positions 1 and 3 in the compounds 3a-f were strongly deshielded $(\delta$ 12.51-10.95) and appeared as singlet in their ${ }^{1} \mathrm{H}$ NMR spectra (Table 3 ). The N-H protons at position 3 in these compounds were found comparatively more deshielded than protons at position 1 .
In some compounds (3c, $\mathbf{3 d} \& \mathbf{3 f}$ ) more deshielding of the $\mathrm{N}-\mathrm{H}$ protons were observed due to presence of thiocarbonyl group. This may be attributed to the greater polarizability of sulfur in comparison to oxygen. 
Table 3. ${ }^{1} \mathrm{H}$ NMR spectral data of the compounds 3a-f. [( $\left.\delta\right)$ in ppm].

\begin{tabular}{|c|c|c|c|c|c|c|c|}
\hline Compound & $3-\mathrm{H}$ & $1-\mathrm{H}$ & Aromatic & $6-\mathrm{H}$ & $5-\mathrm{H}$ & $\mathrm{X}$ & $\mathrm{Y}$ \\
\hline 3a & $\begin{array}{l}11.85 \\
(\mathrm{~s}, 1 \mathrm{H}, \mathrm{N} \underline{\mathrm{H}})\end{array}$ & $\begin{array}{l}10.95 \\
(\mathrm{~s}, 1 \mathrm{H}, \mathrm{N} \underline{\mathrm{H}})\end{array}$ & $\begin{array}{l}8.20-7.20 \\
(\mathrm{~m}, 9 \mathrm{H})\end{array}$ & $\begin{array}{l}6.05 \\
(\mathrm{bs}, 1 \mathrm{H})\end{array}$ & $\begin{array}{l}4.40 \\
(\mathrm{bs}, 1 \mathrm{H})\end{array}$ & $\ldots \ldots$ & $\cdots \cdots$ \\
\hline $3 \mathbf{b}$ & $\begin{array}{l}11.80 \\
(\mathrm{~s}, 1 \mathrm{H}, \mathrm{N} \underline{\mathrm{H}})\end{array}$ & $\begin{array}{l}10.98 \\
(\mathrm{~s}, 1 \mathrm{H}, \mathrm{N} \underline{\mathrm{H}})\end{array}$ & $\begin{array}{l}8.10-7.20 \\
(\mathrm{~m}, 8 \mathrm{H})\end{array}$ & $\begin{array}{l}5.90 \\
(\mathrm{bs}, 1 \mathrm{H})\end{array}$ & $\begin{array}{l}4.40 \\
\text { (bs,1H) }\end{array}$ & & $\begin{array}{l}2.50- \\
2.25 \\
(\mathrm{~m} 3 \mathrm{H}) \\
\left(\mathrm{Ar}-\mathrm{C} \underline{\mathrm{H}}_{3}\right)\end{array}$ \\
\hline $3 c$ & $\begin{array}{l}12.40 \\
(\mathrm{~s}, 1 \mathrm{H}, \mathrm{N} \underline{\mathrm{H}})\end{array}$ & $\begin{array}{l}12.00 \\
(\mathrm{~s}, 1 \mathrm{H}, \mathrm{N} \underline{\mathrm{H}})\end{array}$ & $\begin{array}{l}7.80-7.20 \\
(\mathrm{~m}, 9 \mathrm{H})\end{array}$ & $\begin{array}{l}6.10 \\
(\mathrm{bs}, 1 \mathrm{H})\end{array}$ & $\begin{array}{l}4.50 \\
\text { (bs,1H) }\end{array}$ & & \\
\hline 3d & $\begin{array}{l}12.40 \\
(\mathrm{~s}, 1 \mathrm{H}, \mathrm{N} \underline{\mathrm{H}})\end{array}$ & $\begin{array}{l}12.10 \\
(\mathrm{~s}, 1 \mathrm{H}, \mathrm{N} \underline{\mathrm{H}})\end{array}$ & $\begin{array}{l}7.60-7.20 \\
(\mathrm{~m}, 8 \mathrm{H})\end{array}$ & $\begin{array}{l}5.90 \\
(\mathrm{bs}, 1 \mathrm{H})\end{array}$ & $\begin{array}{l}4.50 \\
(\mathrm{bs}, 1 \mathrm{H})\end{array}$ & & $\begin{array}{l}2.35 \\
(\mathrm{~s}, 3 \mathrm{H}) \\
\left(\mathrm{Ar}-\mathrm{CH}_{3}\right)\end{array}$ \\
\hline $3 \mathbf{e}$ & $\begin{array}{l}11.50 \\
(\mathrm{~s}, 1 \mathrm{H}, \mathrm{N} \underline{\mathrm{H}})\end{array}$ & $\begin{array}{l}11.00 \\
(\mathrm{~s}, 1 \mathrm{H}, \mathrm{NH})\end{array}$ & $\begin{array}{l}8.30-7.30 \\
(\mathrm{~m}, 8 \mathrm{H})\end{array}$ & $\begin{array}{l}6.30 \\
(\mathrm{bs}, 1 \mathrm{H})\end{array}$ & $\begin{array}{l}4.50 \\
\text { (bs,1H) }\end{array}$ & & $\cdots \cdots \cdots$ \\
\hline $3 f$ & $\begin{array}{l}12.51 \\
(\mathrm{~s}, 1 \mathrm{H}, \mathrm{NH})\end{array}$ & $\begin{array}{l}11.95 \\
(\mathrm{~s}, 1 \mathrm{H}, \mathrm{NH})\end{array}$ & $\begin{array}{l}8.30-7.20 \\
(\mathrm{~m}, 8 \mathrm{H})\end{array}$ & $\begin{array}{l}5.70 \\
(\mathrm{bs}, 1 \mathrm{H})\end{array}$ & $\begin{array}{l}4.45 \\
(\mathrm{bs}, 1 \mathrm{H})\end{array}$ & & \\
\hline
\end{tabular}

The proton at position 6 in 3a-f appeared as a broad singlet due to the vicinal coupling with the proton at position 5 . The chemical shifts were observed at $\delta 6.30-5.70$. The $5-\mathrm{H}$ in these compounds gave signals at $\delta 4.50-4.40$ as broad singlet due to the coupling received from the proton at position 5 .

The chemical shifts for the aromatic protons in 3a-f were found in good agreement with the literature values. ${ }^{15,16}$
The structures of the compounds 3a-f were further confirmed by their ${ }^{13} \mathrm{C}$ NMR spectra (Table 4). The chemical shifts of carbonyl carbon at 4-C were found to be deshielded in the range of $\delta 188.47-160.96$. The chemical shifts of 9-C were also deshielded ( $\delta$ 163.48-150.60). This value is comparable with the ${ }^{13} \mathrm{C}$ NMR chemical shifts of cyclohexyl methyl ketone. ${ }^{17}$

Table 4. ${ }^{13} \mathrm{C}$ NMR spectral data of the compounds 3a-f. $[(\delta)$ in ppm]

\begin{tabular}{|c|c|c|c|c|c|c|c|c|c|c|}
\hline $\begin{array}{c}\text { Compoun } \\
\mathrm{d}\end{array}$ & 4-C & $9-\mathrm{C}$ & 7-C & $2-C$ & $\begin{array}{l}\text { Aromatic } \\
\text { carbons }\end{array}$ & $6-C$ & $10-\mathrm{C}$ & $5-C$ & $X$ & $\mathrm{Y}$ \\
\hline $3 a$ & $\begin{array}{l}172.0 \\
8\end{array}$ & $\begin{array}{l}163.4 \\
8\end{array}$ & $\begin{array}{l}154.6 \\
7\end{array}$ & $\begin{array}{l}144.4 \\
7\end{array}$ & $\begin{array}{l}139.89- \\
126.10\end{array}$ & $\begin{array}{l}104.0 \\
2\end{array}$ & $\begin{array}{l}87.5 \\
4\end{array}$ & 35.11 & & \\
\hline $3 b$ & $\begin{array}{l}188.4 \\
7\end{array}$ & $\begin{array}{l}163.3 \\
0\end{array}$ & $\begin{array}{l}154.5 \\
5\end{array}$ & $\begin{array}{l}145.2 \\
3\end{array}$ & $\begin{array}{l}143.74- \\
122.78\end{array}$ & $\begin{array}{l}102.9 \\
8\end{array}$ & $\begin{array}{l}87.0 \\
8\end{array}$ & 34.35 & & $\begin{array}{l}20.80 \\
(\mathrm{Ar}- \\
\left.\mathrm{CH}_{3}\right)\end{array}$ \\
\hline $3 c$ & $\begin{array}{l}160.9 \\
6\end{array}$ & $\begin{array}{l}153.2 \\
3\end{array}$ & $\begin{array}{l}143.9 \\
3\end{array}$ & $\begin{array}{l}173.7 \\
7\end{array}$ & $\begin{array}{l}133.78- \\
125.96\end{array}$ & $\begin{array}{l}104.7 \\
6\end{array}$ & $\begin{array}{l}92.4 \\
5 \\
\end{array}$ & 34.85 & & \\
\hline $3 d$ & $\begin{array}{l}160.9 \\
9\end{array}$ & $\begin{array}{l}158.4 \\
7\end{array}$ & $\begin{array}{l}146.0 \\
0\end{array}$ & $\begin{array}{l}183.0 \\
1\end{array}$ & $\begin{array}{l}142.94- \\
123.25\end{array}$ & $\begin{array}{l}105.7 \\
5\end{array}$ & $\begin{array}{l}92.1 \\
3\end{array}$ & 34.28 & & $\begin{array}{l}20.80 \\
(\mathrm{Ar}- \\
\left.\mathrm{CH}_{3}\right)\end{array}$ \\
\hline $3 e$ & $\begin{array}{l}163.2 \\
1\end{array}$ & $\begin{array}{l}150.6 \\
0\end{array}$ & $\begin{array}{l}147.4 \\
4\end{array}$ & $\begin{array}{l}143.5 \\
2\end{array}$ & $\begin{array}{l}131.26- \\
123.93\end{array}$ & $\begin{array}{l}108.2 \\
2\end{array}$ & $\begin{array}{l}87.1 \\
2\end{array}$ & 34.59 & & \\
\hline $3 f$ & $\begin{array}{l}163.1 \\
1\end{array}$ & $\begin{array}{l}150.7 \\
5\end{array}$ & $\begin{array}{l}146.8 \\
0\end{array}$ & $\begin{array}{l}173.8 \\
9\end{array}$ & $\begin{array}{l}140.89- \\
126.13\end{array}$ & $\begin{array}{l}105.0 \\
8\end{array}$ & $\begin{array}{l}92.8 \\
1\end{array}$ & 34.62 & & \\
\hline
\end{tabular}

In the compounds $\mathbf{3 a}, \mathbf{3 b}$ and $\mathbf{3 e}$, the chemical shifts of carbonyl carbons at 2-C were found to be at $\delta 145.23$ 143.52 and are relatively less deshielded due to the resonance of amide functional group. In the compounds 3c, 3d and 3f, the chemical shifts of thioxo carbon at 2-C were found to be at $\delta 183.01-173.77$. This explains that the replacement of a carbonyl group by a thiocarbonyl group results in a downfield shift. ${ }^{19,20}$

The chemical shift values for $7-\mathrm{C}$ and $6-\mathrm{C}$ in these compounds were observed at $\delta$ 154.67-143.93 and $\delta$ 108.22-102.98, respectively. The $10-\mathrm{C}$ of the compounds

showed chemical shift values at $\delta 92.81-87.08$ which were comparable to the earlier report ${ }^{14}$ of the ${ }^{13} \mathrm{C}$ NMR spectral data of the monosubstituted barbiturates at 10-C. The chemical shift values for 5-C in these compounds were observed at $\delta$ 35.11-34.28.

The ${ }^{13} \mathrm{C}$ NMR chemical shifts for the carbons of aromatic rings were assigned on the basis of a correlation chart available in the literature. ${ }^{18}$

The compounds 3a-f showed peaks for their respective molecular ions $\left(\mathrm{M}^{+}\right)$with sodium in their high resolution mass spectra at $\mathrm{m} / \mathrm{z} 375.7708$ (22.50\%), 389.6605 (9\%), 391.0455 
(12\%), 405.8605 (11.11\%), $420.7580(6 \%)$ and 436.5430 $(10 \%)$ respectively. The isotopic pattern for $\mathrm{Cl}$ atom $\left({ }^{35} \mathrm{Cl} /{ }^{37} \mathrm{Cl}, 3: 1\right)$ was observed in the molecular mass of the compounds 3a-f. The $\mathrm{M}^{+}+2$ with $\mathrm{Na}$ were observed at 377.2050 (7.50\%), 391.7650 (3\%), 393.0450 (4\%), 407.0180 (3.7\%), $422.3205(2 \%)$ and 438.5033 (3.25\%) respectively.

\section{Acknowledgement}

Authors gratefully acknowledge the help of Prof. Teruo Shinmyozu, Department of Molecular Chemistry, Kyushu University, Fukuoka, Japan for recording mass spectra and determining elemental analyses of our compounds. Our special thanks goes to Prof. Takashi Sugimura, Graduate School of Material Science, University of Hyogo, Japan for recording proton and carbon-13 nmr spectra of the compounds.

\section{References}

1. Senda, S., H. Fujimura and H. Izumi, 1968. Barbituric acid analgesics, Japan Patent, 193, 6824.

2. Levitt, G., 1982. Herbicidal sulfonamides, US Patent 4339267.

3. O'Callaghan, C. N. and M. L. Conalty, 1983. Anticancer Agents: XVII. Synthesis and antitumour activity of 2-ary14-oxo-2,3-dihydrobenzopyrano[2,3-d] pyrimidines, and 4substituted 2-aryl-5h-benzopyrano-[2,3-d] pyrimidines, Proc. R. Ir. Acad., 83B, 241

4. Wrigglesworth, R.,W. D. Inglish, D. B. Livingstone, C. J. Suekling and H. C. S. Wood, 1984. Specific enzyme inhibitors in vitamin biosynthesis. Part 6. Identification of an affinity chromatography ligand for the purification of riboflavin synthase, J. Chem. Soc. (Perkin Trans I), 5, 959963.

5. Rao, A. S. and R. B. Mitra, 1974. Synthesis of heterocycles. II. Pyrano[2,3-d]pyrimidines, Ind. J. Chem., 12, 1028; Chem Abstr, 1975, 82, 112023.

6. Junek, H.and H. Aigner, 1973. Synthesen mit Nitrilen, XXXV. Reaktionen von Tetracyanäthylen mit Heterocyclen. Chem Ber, 106, 914-921.

7. Noboru, S., K. Yoshikazu and T. Psurematsu, 1973. Chem Pharm Bull, 21, 2639.

8. Bararjanian, M., S. Balalaie, B. Movassagh and A. M. Amani, 2009. One-Pot Synthesis of Pyrano[2,3-d]pyrimidinone Derivatives Catalyzed by L-Proline in Aqueous Media, J. Iran. Chem. Soc., 6(2), 436-442.
9. Ahlwalia, V. K., R. Aggarwal and R. Kumar, 1993. A Convenient One-Pot Synthesis of 5-Aryl-7-methyl-1,2,3,4tetrahydro-2, 4-dioxo-5H-pyrano(2,3-d) pyrimidines, Ind. $J$. Chem., 32B, 963-964.

10. Ahmed, M. G., U. K. R. Romman, S. M. Ahmed, K. Akhter, M. E. Halim and M. Salauddin, 2006. A study on the synthesis of 5,7-diaryl-1,2,3,4-tetrahydro-2,4-dioxo-5 $\mathrm{H}$-pyrano[2,3d]pyrimidines; Bangladesh J. Sci. Ind. Res. 41(3-4), 119-128 and references herein.

11. Ahmed, M. G., U. K. R. Romman, K. Akhter, M. E. Halim, M. M. Rahman and S. M. Ahmed, 2011. A one-step synthesis of 5,7-Diaryl-1,5-dihydro (or 1,2,3,5-tetrahydro)-pyrano[2,3d] pyrimidin-2,4-diones (or 2-thioxo-4-ones), Ind. J. Chem. 50B, 946-948.

12. Kharchenko, V. G., L. I. Markova and K. M. Korshunova, 1976. On Reactions of oxo-1, 5-diketones with sulfurous reagents, $Z h$. Org. Khim., 12(3), 663; Chem Abstr, 1976, 85, 32775c.

13. Vogel, A. I. A Text Book of Practical Organic Chemistry, $4^{\text {th }}$ edition, Longman Group Ltd., London, p. 796.

14. Bojarski, J. T., J. L. Mokrosz, H. J. Barton and M. H. Paluchowaka, 1985. Advances in Heterocyclic Chemistry (Review Article), 38, 229-297.

15. Silverstein, R. M., G. C. Bassler and T. C. Morill, 1991. Spectroscopic Identification of Organic Compounds, $5^{\text {th }}$ edition, John Wiley \& Sons, N.Y..

16. Kemp, W. 1991. Organic Spectroscopy, $3^{\text {rd }}$ edition, Macmillan, London.

17. Marr, D. H. and J. B. Stothers, $1965 .{ }^{13}$ C NMR Studies: Part VI. Carbon-13 Spectra of $\alpha, \beta$ - Unsaturated Carbonyl Compounds, Canad. J. Chem., 43, 596-607.

18. Levy, G. C. and G. L. Nelson, 1972. Carbon-13 Nuclear Magnetic Resonance for Organic Chemists, John Wiley \& Sons, N. Y..

19. Otto, H. H. and J. Triepel, 1976. Synthesis and structure of 7,11diphenyl-2,4-diazaspiro[5.5] undecan-1,3,5,9-tetraones, I. Liebigs Ann. Chem., 1982-1991.

20. Ahmed, M. G., S. A. Ahmed, S. M. Ahmed, A. Hussam and M. M. Hossain, 2005. Synthesis of 7,11-diaryl-2,4-diazaspiro[5,5] undecane-3-oxo(or thioxo)-1,5,9-trines, Part-1. J. Chem. Res., 10, 622-625. 